Research Article

\title{
On the Analysis of the Contact Conditions in Temporomandibular Joint Prostheses
}

\author{
B. Gonzalez-Perez-Somarriba, ${ }^{1}$ G. Centeno ${ }^{D},{ }^{1}$ C. Vallellano, ${ }^{1}$ and \\ L. M. Gonzalez-Perez ${ }^{2}$ \\ ${ }^{1}$ Department of Mechanical and Manufacturing Engineering, University of Seville, Seville, Spain \\ ${ }^{2}$ Department of Oral and Maxillofacial Surgery, Virgen del Rocío University Hospital, Seville, Spain
}

Correspondence should be addressed to G. Centeno; gaceba@us.es

Received 22 January 2018; Accepted 14 March 2018; Published 22 April 2018

Academic Editor: Antonio Riveiro

Copyright $(2018$ B. Gonzalez-Perez-Somarriba et al. This is an open access article distributed under the Creative Commons Attribution License, which permits unrestricted use, distribution, and reproduction in any medium, provided the original work is properly cited.

Temporomandibular joint replacement (TMJR) is a complex surgical procedure in which the artificial joints available must assure the anatomical reconstruction and guarantee a good range of the natural temporomandibular joint (TMJ) movements. With this aim, different types of TMJ prostheses, including the stock prosthetic system and custom-made prostheses, are being currently implanted. Although temporomandibular joint replacements (TMJRs) are expected to accomplish their function during a number of years, they might actually fail and need to be replaced. This paper analyzes different design factors affecting the contact stress distributions within the TMJ prosthesis interface, which are consequently involved in their deterioration and final failure of the prosthetic device. With this purpose, a numerical model based on finite elements has been carried out in order to evaluate the stress states attained in different prosthesis configurations corresponding to general types of TMJ prostheses. On the other hand, the actual degradation of resected implants has been evaluated via optical microscopy. The linkage between the numerical simulations performed and experimental evidence allowed the authors to establish the different wear and damage mechanisms involved in the failure of stock TMJ prostheses. Indeed, the results obtained show that the contact stresses at the interface between the mandible and the glenoid fossa components play a key role in the failure process of the TMJR devices.

\section{Introduction}

Medical prostheses are artificial devices used to replace the main function of damaged human tissues. This damage can occur as a result of degenerative diseases, traumatic accidents, or tumours. Besides, there are a variety of reasons promoting the intensive use of prostheses nowadays including the earlier diagnosis of diseases, the greater rate of success in surgical procedures, the higher longevity of the population, a demand for better quality of life, and the more active lifestyles, among others. As a consequence, for the last few decades, the scientific community and industrial companies have been focusing on the study and development of a new kind of prosthesis [1] especially for bones and their joints [2,3], mainly based on the use of groundbreaking manufacturing technologies such as additive manufacturing $[4,5]$ or other innovative machining technologies [6] and forming processes [7-9]. These approaches have been carried out from different points of view including medical, engineering, and industrial perspectives, being the principles of multidisciplinary approaches essential for the final purpose of the design and the manufacturing of even more successful devices to be implanted.

Regarding cranial, craniofacial, and maxillofacial prostheses, they have been applied clinically for over 20 years [10] as the most successful procedure for reconstruction of these tissues once they are irreversibly damaged. As occurs with other kinds of prostheses, they are currently being implanted at a significant increasing rate and consequently applied to a wider age range of patients. In the case of maxillofacial prostheses, TMJR makes use of complex devices with the aim of providing a biomechanical solution to advanced-stage TMJ disease [11]. Although they are designed and manufactured in 


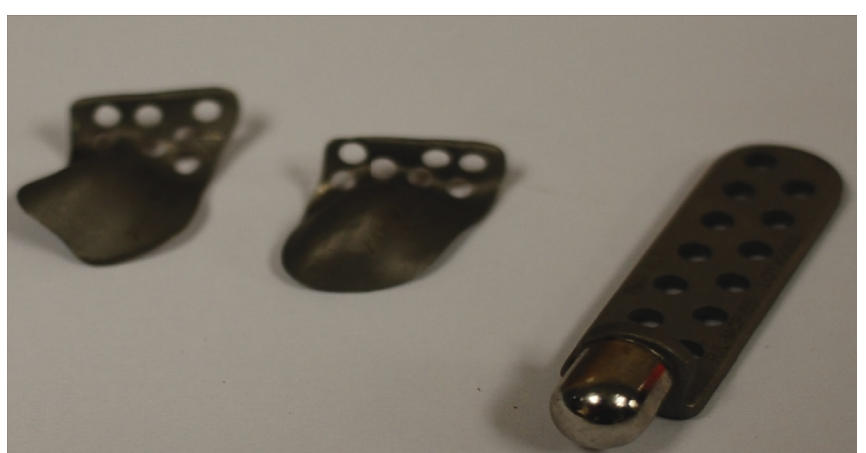

(a)

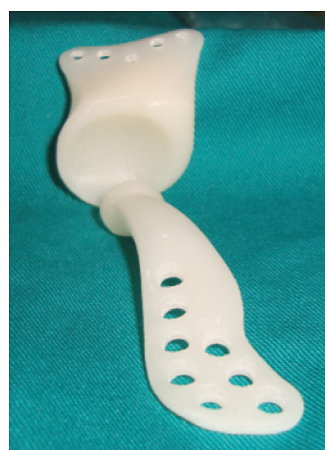

(b)

Figure 1: Stock and custom-made prostheses: (a) metal-on-metal (Cr-Co) Christensen ${ }^{\circledR}$ prosthesis including 2 geometries of the glenoid fossa component and (b) customised Biomet-Lorenz ${ }^{\circledR}$ prosthesis model on resin for evaluation prior to the surgical procedure.

order to accomplish their function during a number or years, they might actually fail and need to be substituted [12, 13], resulting in repetitive surgical procedures for the patients and the increase of medical costs. Failure of TMRJs is a consequence of the extremely challenging physical environment into which the prosthesis is implanted, being degradation, friction in the joint contact interface, tribocorrosion [14], and the subsequent wear the main causes which are most commonly associated with this failure.

Two main categories of TMJR devices are commonly implanted: stock prostheses, which the surgeon must fit at implantation, and custom-made prostheses. One of the most important examples of stock TMJ prostheses is the metal-onmetal (Cr-Co) Christensen implant, whose behaviour has been analyzed not only from medical [15] but also from engineering view, as for instance carrying out numerical modelling using finite element analysis [16]. Currently, there exist 44 models depending on different sizes and dimensions for the particular configuration of the Christensen implant. On the other hand, customised TMJR devices can be represented by the ultrahigh-molecular weight polyethylene (UHMWPE) on metal (Cr-Co) Biomet Microfixation TMJ Replacement System $^{\circledR}$ [12], being the glenoid fossa cup made of UHMWPE and the mandibular condyle of $\mathrm{Cr}$-Co. Figure 1 depicts the 2 mentioned TMJR types, whereas the main characteristics and differences between stock and custom-made TMJ prostheses can be found in previous research works as [17].

Taking into account this background, the use of suitable biomaterials [18] and numerical methods [19, 20] for increasing knowledge of stress/strain states in the TMJ prosthesis interface should lead to the correct design parameters [21] that could decrease material wear and increase the longevity of joint replacement devices.

In this scientific perspective, this paper analyzes different design factors affecting contact stresses at the TMJ prosthesis interface (i.e., the contact interface between the mandibular and the glenoid fossa components), which are consequently involved in their deterioration and final failure. With this purpose, a numerical model and the subsequent finite element analysis (FEA) have been carried out in order to evaluate the stress states attained in different prosthesis configurations. On the other hand, the actual degradation of replaced implants has been evaluated by optical microscopy in order to establish a connection between FE numerical simulations and experimental evidence. The results show that the contact stresses in the interface between the mandibular and the glenoid fossa components play a key role in the failure process of the TMJR devices.

After contextualizing this research within the state of the art in TMJR, Section 2 presents the methodology for obtaining the inputs variables for the numerical simulations, whereas Section 3 focuses on the FEA. Section 4 discusses the numerical results obtained and their linkage with the actual degradation of real replaced implants. The contributions of the paper are exposed in Section 5 .

\section{Methodology}

This section exposes the methods used for providing the inputs that are necessary for carrying out numerical simulations using a finite element code. In general, in what concerns the analysis, design, and/or manufacturing of medical devices, a precise methodology must be established. Indeed, the previous work of the authors has dealt with this issue $[8,22]$ proposing well-defined road maps including every possible flow of materials, processes, and information.

Analogically, in the current case, the methodology starts with the processing of a patient's computerized axial tomography (CAT) provided by a DICOM (Digital Imaging and Communications in Medicine) standard file. Muscles, tendons, and other remaining tissues have to be deleted in order to isolate just the bones. As depicted in Figure 2, the results of this procedure have to be transferred to a computer-aided design (CAD) software, which in this occasion was the platform CATIA ${ }^{\circledR}$. The processing of the DICOM files for obtaining the selected geometries corresponding to the cranial bones was carried out using the open-source software InVesalius ${ }^{\circledR}$ for reconstruction of computed tomography and magnetic resonance images. Finally, the CAD file containing the configuration of the mandibular geometry considered is exported to the finite element program Abaqus. 


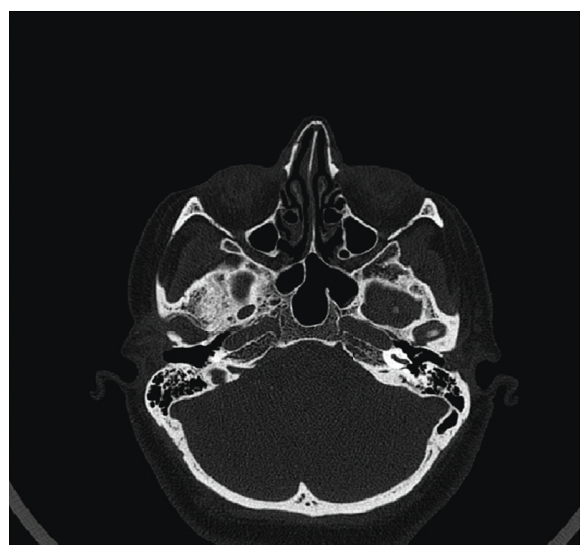

(a)

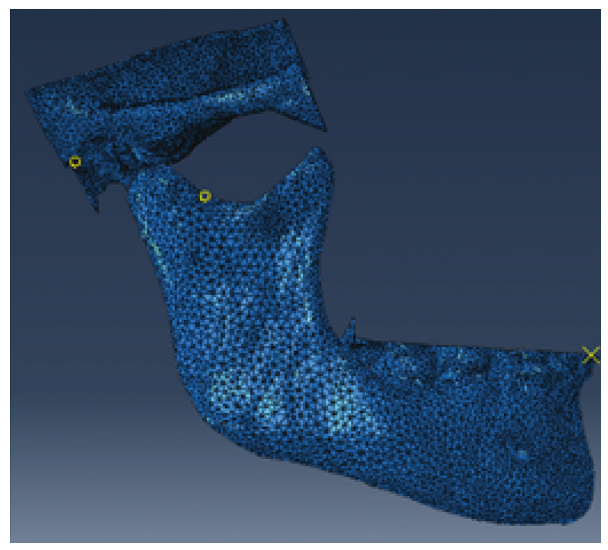

(b)

FIgURE 2: From DICOM to CAD: (a) the patient's computerized axial tomography (CAT) cut by a horizontal plane and (b) final configuration of the selected geometry in Abaqus ${ }^{\circledR}$.

TABLE 1: Cases of the study considered for the FEA.

\begin{tabular}{lccc}
\hline Prosthesis components & $\begin{array}{c}\text { Generic glenoid fossa } \\
\text { component }\end{array}$ & $\begin{array}{c}\text { Biomet }{ }^{\circledR} \text { glenoid fossa } \\
\text { component }\end{array}$ & $\begin{array}{c}\text { Christensen glenoid fossa } \\
\text { component }\end{array}$ \\
\hline $\begin{array}{l}\text { Christensen mandibular } \\
\text { component }\end{array}$ & Case 1 & Similar to the generic case & Christensen; Ramos and Mesnard \\
Biomet mandibular component & Case 2 & Bimilar to real Biomet \\
Case 3 & Biomet & Not proceeded \\
\hline
\end{tabular}

On the other hand, the TMJ prostheses present two parts, the glenoid and the mandibular components, working as the human joint as they are connected through an almost spherical interface (Figure 1) with different radii (notice that mandibular component radius is slightly smaller) and hold together by a series of mandibular muscles.

In this regard, 3 different cases of the study have been considered for the numerical evaluation of the contact stresses at the prosthesis interface, that is, at the contact surface between the glenoid fossa and the mandibular components. As shown in Table 1, the three cases are defined as follows: (i) "case 1" has a real Cr-Co Christensen mandibular component combined with a generic Cr-Co glenoid fossa component, (ii) "case 2 " is geometrically similar to "case 1" but considered a generic UHMWPE glenoid fossa component, and (iii) "case 3" uses a Cr-Co Biomet-type mandibular component (higher spherical radius at the TMJ prosthesis interface) combined with a generic UHMWPE glenoid fossa component. The illustration of these cases is depicted in Table 2.

As mentioned above, these three cases were numerically analyzed under the light of finite element simulations and experimentally evaluated by measuring the damaged areas on the TMJ prosthesis contact surface via optical microscopy. The procedures followed and the results obtained are discussed in the following sections.

\section{Finite Element Analysis}

The FEA was carried out using the implicit solid-formulation finite element method software Abaqus [22]. The simulation was performed considering elastic behaviour of all the materials involved and considering isotropic behaviour of the cortical bone and TMJ prosthesis component, either made of metal (Cr-Co) or polymer (UHMWPE). The material properties of Cr-Co, UHMWPE, and the cortical bone provided in Table 3 were taken from the literature [16]. These values are similar to others provided in different research studies [23, 24].

Once the assembly of TMJ prostheses fixed to the mandibular bone is performed (Figure 3(a)), all the bodies, both the mandibular cortical bone and the two TMJ prosthesis components, were meshed using the 4-node tetrahedron C3D4 [22]. The mesh size was set to $0.8 \mathrm{~mm}$ and refined until $0.16 \mathrm{~mm}$ as approaching to the TMJ prosthesis contact interface, as can be seen in Figure 3(b). In this regard, a sensitivity analysis was performed until establishing the optimal size of the mesh at the critical zones, especially at the contact interface up to the convergence of contact stress distribution. Besides, surface-to-surface contact conditions were implemented at the interface, establishing "hard contact" based on augmented Lagrange for the normal direction and a penalty algorithm for the tangential direction, considering Coulomb's friction conditions with the friction coefficient provided in Table 4 [25] for the two material configurations considered, that is, metal on metal (case 1 ) and polymer on metal (cases 2 and 3 ), which were set to 0.22 and 0.06 , respectively.

Regarding the boundary conditions (shown in Figure 4), the glenoid fossa component is considered to be fully constrained at the 5 holes (where titanium screws should fix the component to the cranial bone), and the vertical displacement 


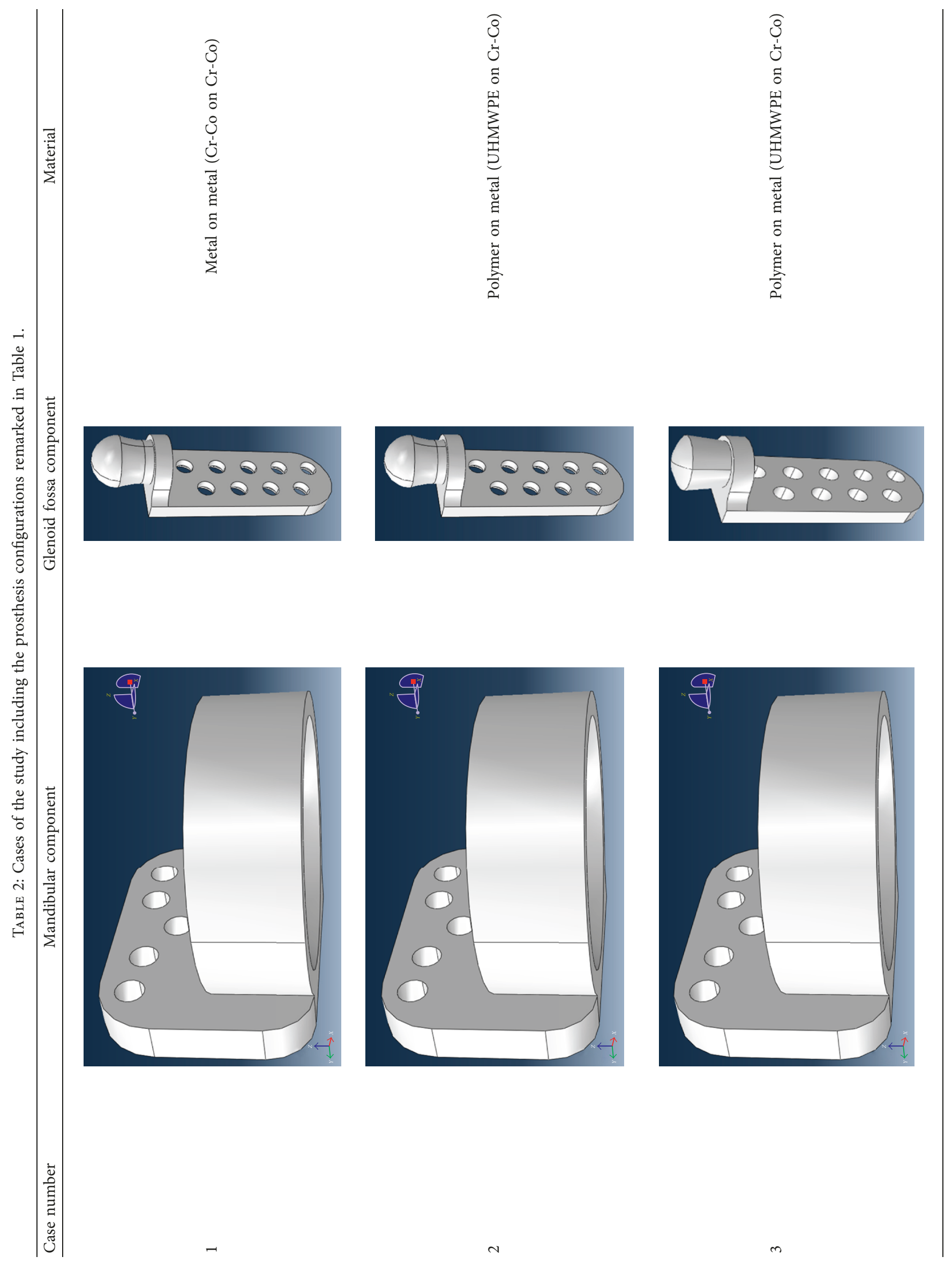


TABLE 3: Elastic mechanical properties of the materials involved in the FEA [16].

\begin{tabular}{lccc}
\hline Material & Elastic modulus $E(\mathrm{GPa})$ & Poisson's ratio $v$ & Yield stress $\sigma_{\mathrm{y}}(\mathrm{MPa})$ \\
\hline UHMWPE & 0.7 & 0.35 & 28 \\
Cr-Co & 210 & 0.3 & 550 \\
Cortical bone & 14.7 & 0.28 & $*$ \\
\hline
\end{tabular}

*Yield stress of the cortical bone varies depending on the zone and is irrelevant for the study of the TMJR.

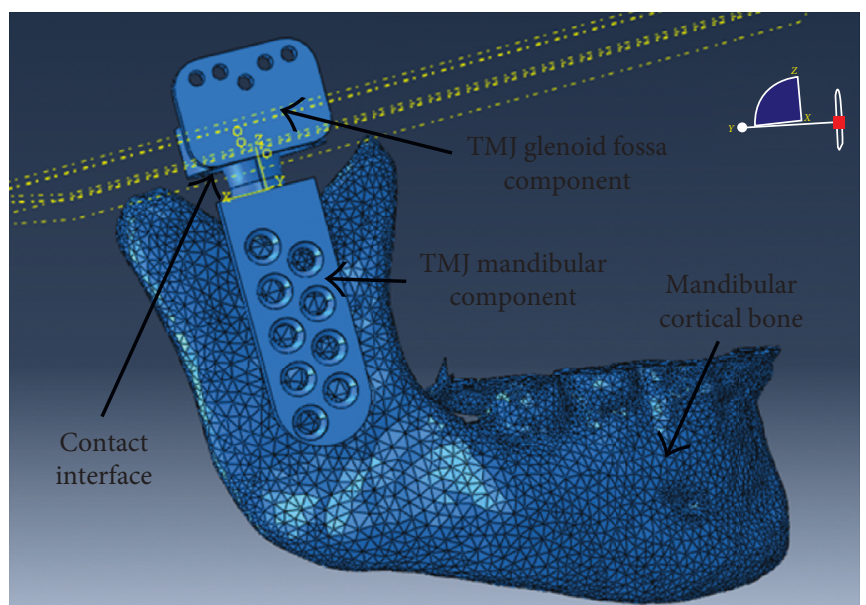

(a)

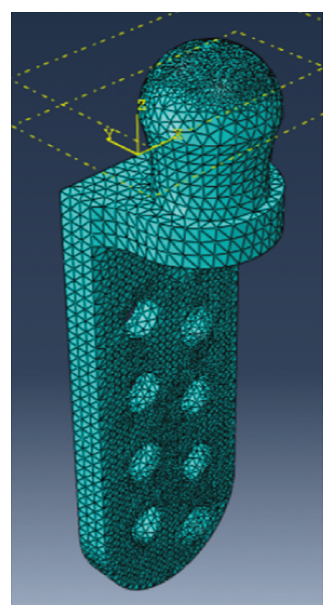

(b)

Figure 3: (a) Assembly of TMJ prostheses fixed to the right half of the mandibular bone and (b) meshing of the TMJ mandibular component. Notice the mesh refinement at the contact interface.

TABle 4: Coulomb's friction coefficients for different prosthetic contact pairs [25].

\begin{tabular}{lc}
\hline Contact pair & Friction coefficient \\
\hline Cr-Co on Cr-Co & 0.22 \\
UHMWPE on Cr-Co & 0.06 \\
\hline
\end{tabular}

of the superior circular surface is also constrained. The cranial bone is not considered in the analysis as far as from a medical point of view; from the inspection of this zone, there are no appreciable damage $[13,17]$ due to the correct prosthesis biointegration usually attained after surgical procedure. In this sense, it must also be noticed that the temporal component of the prosthesis allows the condyle rotations but limits the (natural) translations with respect to the cranium. On the other hand, the TMJ mandibular component is tied to the mandibular bone through its projected surface. Finally, symmetry conditions are assumed in the middle vertical plane cutting the mandibular bone in its left and right halves.

The intensities of the different loads applied to the corresponding muscles (deep and superficial masseters, medial pterygoid, temporalis, and medial temporal), exerted during the real human mastication cycle, have been determined by Mesnard et al. in a previous research work [20]. In the current study, the force vectors applied at the corresponding muscle locations have been set for a mouth opening of $15 \mathrm{~mm}$ according to the values provided by Ramos and Mesnard [16]. It must be noticed that these loads were applied at their specific locations using multipoint constrains (MPCs) connecting a series of nearby elements to a rigid point with the aim of avoiding punctual stress concentrators.

Finally, it is worth mentioning that the overall central processing unit (CPU) time for the 3 cases analyzed was between 1 and 2 hours on a laptop using one Intel i7-6500U CPU $(2.60 \mathrm{GHz})$ processor.

\section{Results and Discussion}

In the first part of this section, an experimental assessment of TMJ prostheses resected from patients has been carried out in order to evaluate the actual degradation of the implants and the size of the damaged area. The second part presents the numerical results provided by the FEA. Finally, using the connection between the numerical results and the experimental evidence, the influence of interface contact stresses on the possible failure mechanisms of TMJ prosthesis is discussed.

4.1. Experimental Evaluation of Replaced TMRJ. As exposed above, a series of Christensen TMJ prostheses resected from the patient after the evidence of wrong behaviour were examined via optical microscopy in order to evaluate the different regions affected by wear. The measurements were performed in these regions using a Nikon SMZ800 stereomicroscope with $\times 10$ to $\times 60$ magnification and analyzed using KAPPA Image Base Metreo 2.7.2. In this regard, Figure 5 depicts the damaged regions for a specimen of reference, consisting in an almost circular damaged area at the mandibular component that seems to be affected by a combination of plastic deformation and wear, and a horizontally elongated 


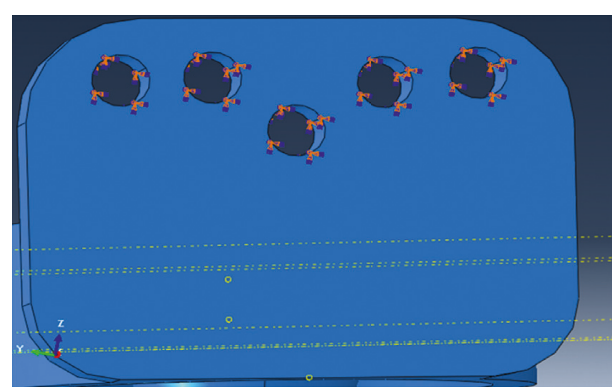

(a)

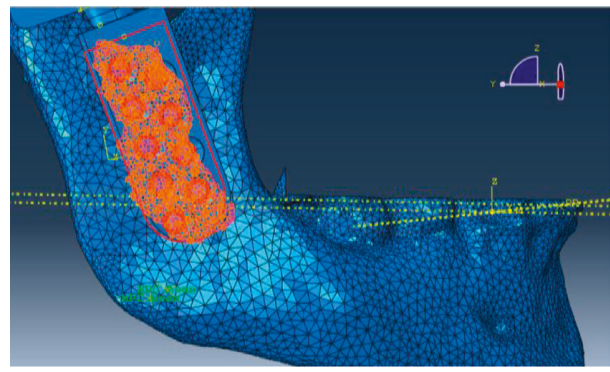

(c)

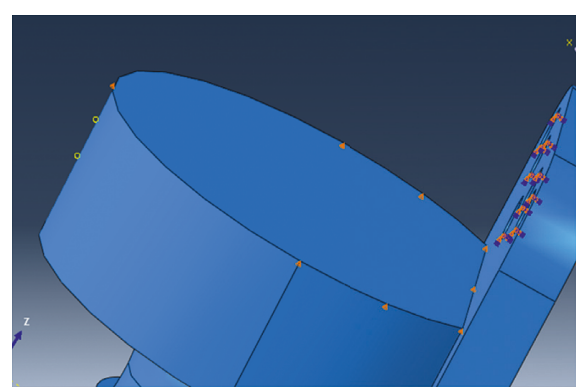

(b)

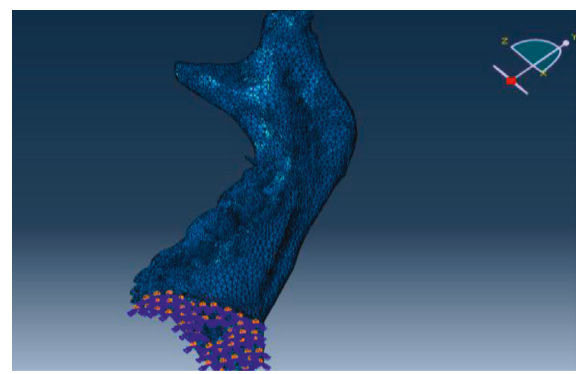

(d)

FIGURE 4: Boundary conditions: (a) glenoid fossa component fully constrained at the cranium bone fixation (b) and vertical constrained at the contact with glenoid fossa. (c) Mandibular component tied to the mandibular bone. (d) Symmetry conditions.

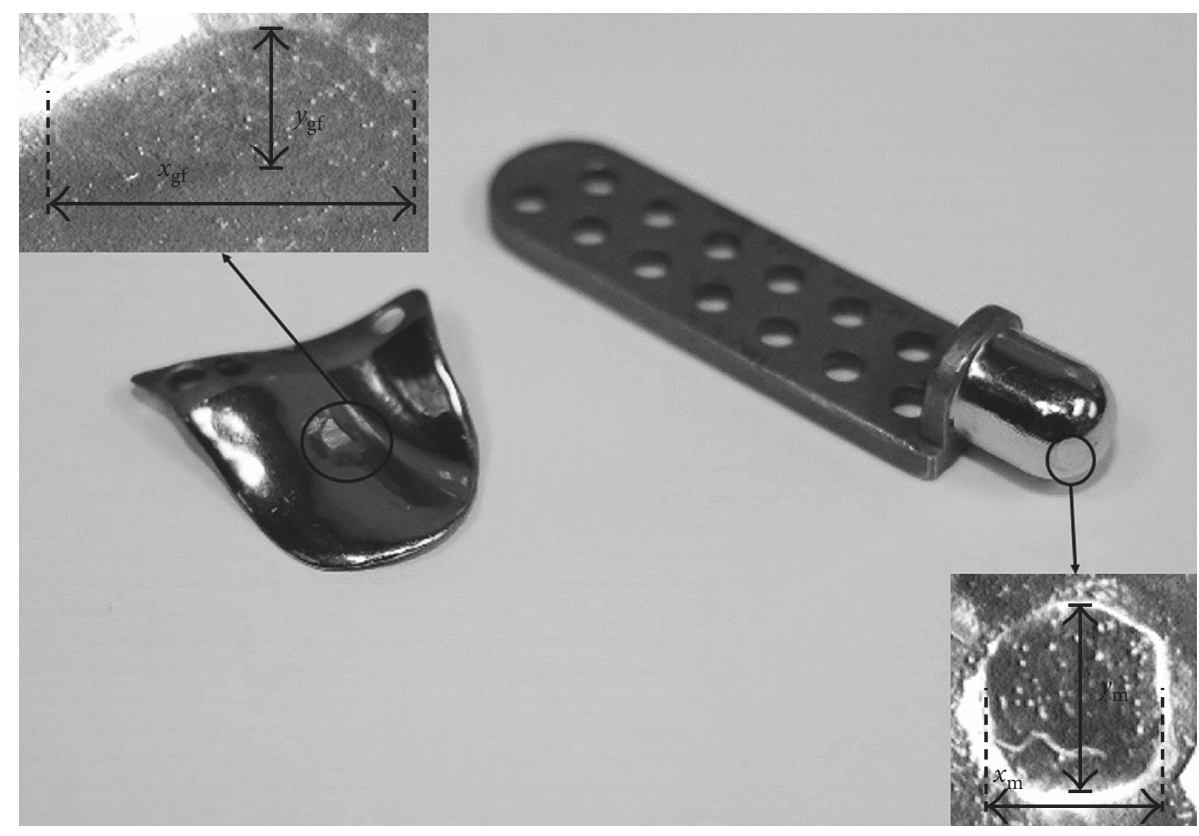

FIGURE 5: Evaluation of the damaged zone within the contact interface of a resected after failure Christensen TMJ prosthesis specimen by microscope.

damaged zone at the glenoid fossa component that gives the impression of being mainly affected by wear. The average values of these damage zone dimensions are given in Table 5, where $x_{\mathrm{gf}}$ and $y_{\mathrm{gf}}$ correspond, respectively, to the horizontal and vertical dimensions of the damaged zone at the glenoid fossa component, whereas $x_{\mathrm{m}}$ and $y_{\mathrm{m}}$ are the dimensions at the mandibular component.

It must be noticed that the evaluation of the damaged zone within the contact area at the glenoid fossa component seems to be related to the action of fluctuating contact loads. These loads produce a time variable surface contact stress field and relative displacement between mating surfaces. Both things promote damage phenomena like tribocorrosion [14], fretting [26, 27], and/or fretting wear [28]. Indeed, this wear-related mechanism would be consistent with the surface experimental observation of the resected prostheses provided by the evaluation by microscope shown in Figure 5. On the contrary, the damaged area at the mandibular TMJ 
TABle 5: Average longitudinal and traverse dimensions of the damaged zone within the contact interface of a resected after failure Christensen TMJ prosthesis specimen by microscope.

\begin{tabular}{llr}
\hline Component & & Damaged zone length $(\mathrm{mm})$ \\
\hline \multirow{2}{*}{ Glenoid fossa component } & $x_{\mathrm{gf}}$ & 5.01 \\
& $y_{\mathrm{gf}}$ & 1.85 \\
Mandibular component & $x_{\mathrm{m}}$ & 2.71 \\
& $y_{\mathrm{m}}$ & 2.73 \\
\hline
\end{tabular}

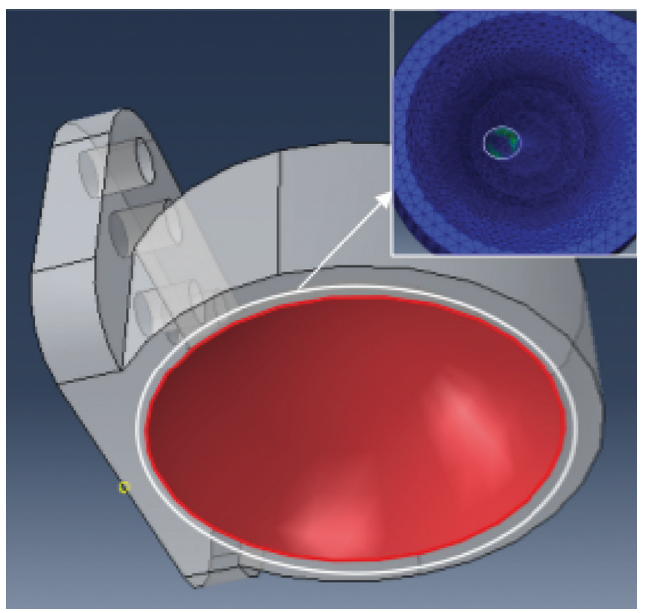

(a)

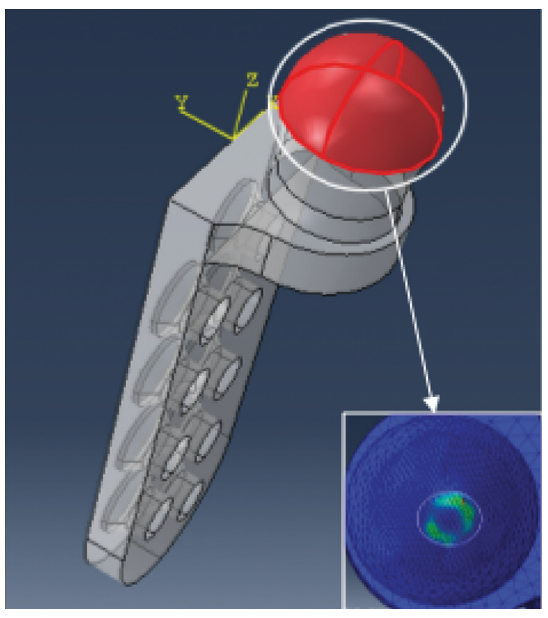

(b)

Figure 6: Evaluation of contact stresses at (a) the glenoid fossa and (b) mandibular components.

component is slightly smaller and has a distinctive circular shape (see the indicated location at the detailed view shown in Figure 6 and the corresponding values in Table 5), presenting this circular damaged area a flat surface changing the radius of this hemispherical contact element. This flat might not only be explained by wear under elastic regime but also can be attributable to a permanent deformation due to an elastic-plastic behaviour in this area.

4.2. Numerical Results. The simulations were carried out using the software Abaqus following the procedure presented in Section 3 for the three cases of the study depicted in Table 2. In every case, the level of contact stresses is evaluated on the contact interface of the TMJ prosthesis at the glenoid fossa and mandibular components (Figure 6).

In this regard, Figure 7 shows the distribution of von Mises contact stresses at the TMJ contact interface for these 3 cases considered (Table 2), providing details of these contact stresses' contour within the contact interface at the glenoid fossa and mandibular components for cases 1 (Figure 7(a)), 2 (Figure 7(b)), and 3 (Figure 7(c)), respectively.

Related to the 3 cases depicted in Figure 7, Table 6 provides the maximum value of equivalent Mises stresses within the contact interface. In this regard, an average of the contact stresses for the contact region has also been obtained in order to provide mean values within this region that can be compared with the material properties.

4.3. Discussion. This section aims at linking the experimental evaluation of resected TMJ prostheses carried out by microscope (presented in Section 4.1) with the numerical results obtained from the FEA in Section 4.2.

In Figure 7(a), the results correspond to case 1, that is, a metal-on-metal TMJ prosthesis similar to the Christensen configuration. The maximum local contact equivalent stress values obtained for the 2 components are in this case sensibly above the average ones. This might be caused by an excessive penetration in the contact algorithm. Besides, it must be noticed that the generic glenoid fossa component is thicker than that in a typical Christensen device, and then, the level of contact stresses is slightly higher than expected. However, the contact stresses obtained are in good agreement with the results by Ramos and Mesnard [16]. In this case, the field of contact stresses is in the order of magnitude of the material yield stress. Therefore, these high values of stresses in conjunction with the examination by microscope of resected Christensen TMJ prostheses carried out in Section 4.1 allow concluding that permanent deformation due to an elastic-plastic behaviour is highly likely in this area. Indeed, it seems that the circular damaged area contending a flat surface in the mandibular component might not be explained only by a wear phenomenon due to tribocorrosion and/or fretting wear under elastic regime.

As can be seen in Figure 7(b), the results corresponding to case 2 (polymer on metal) show a more uniform distribution of contact equivalent stresses but still with high peak values in comparison with the calculated average stresses. However, in this case, there exists a significant region at the glenoid fossa component attaining values of contact stresses above the yield stress of UHMWPE. These results allow concluding that it would not be possible to combine stock metal mandibular 


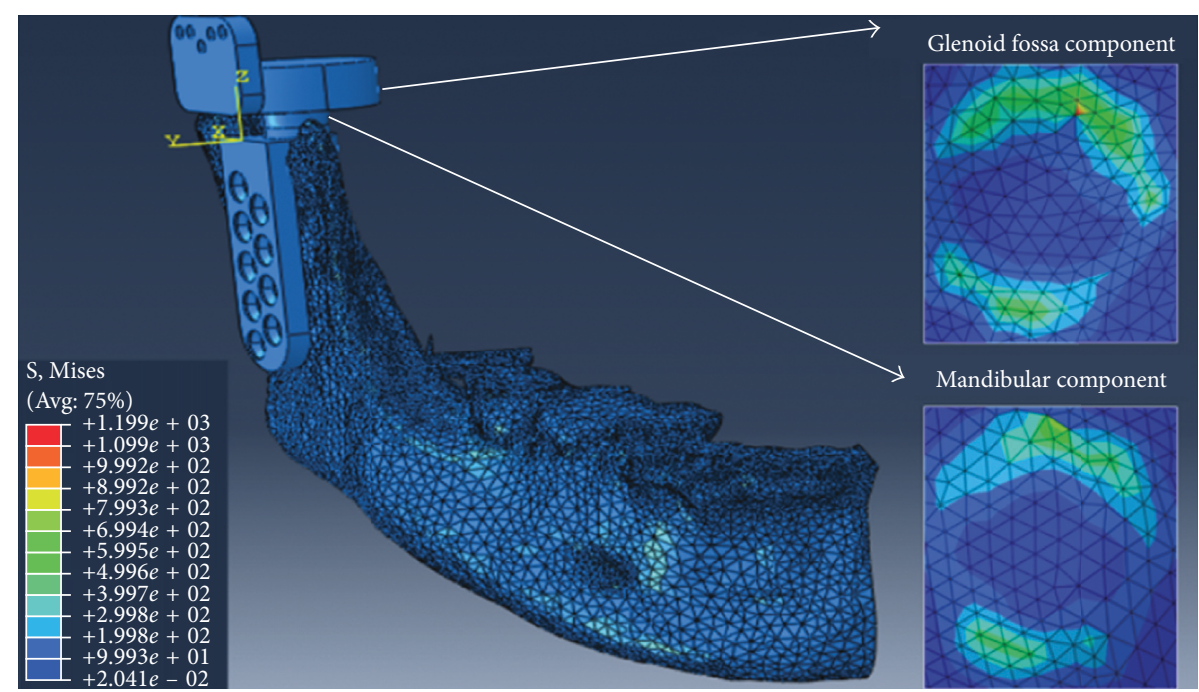

(a)

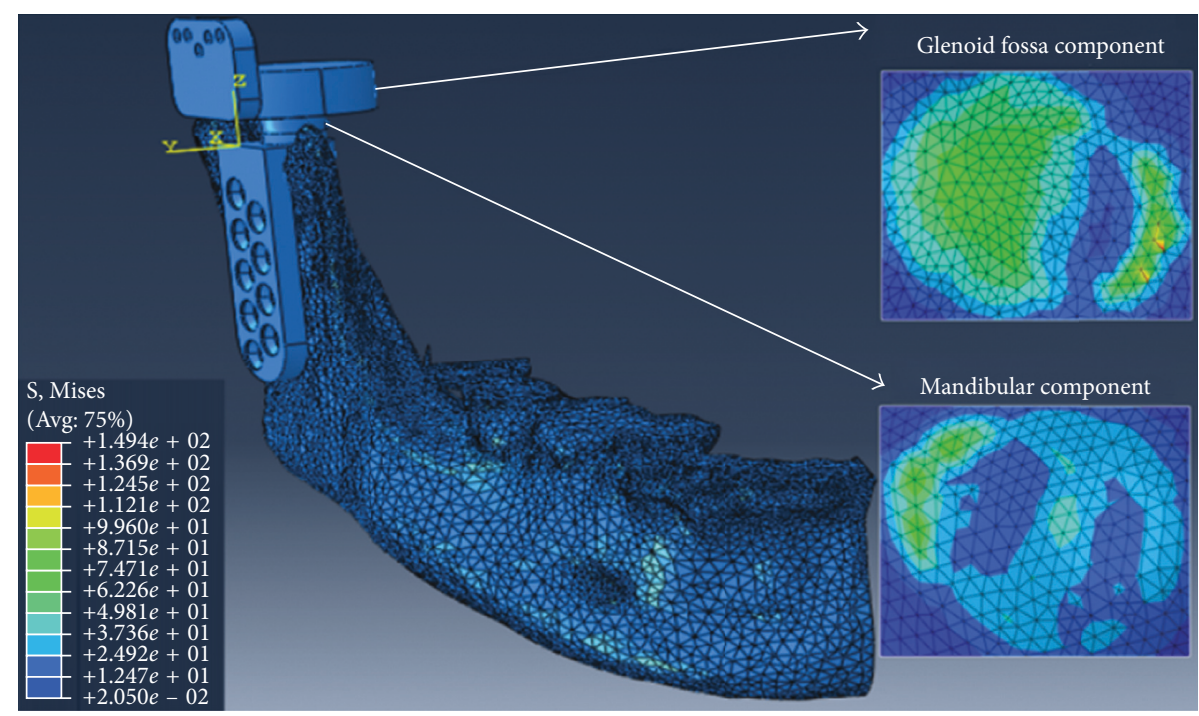

(b)

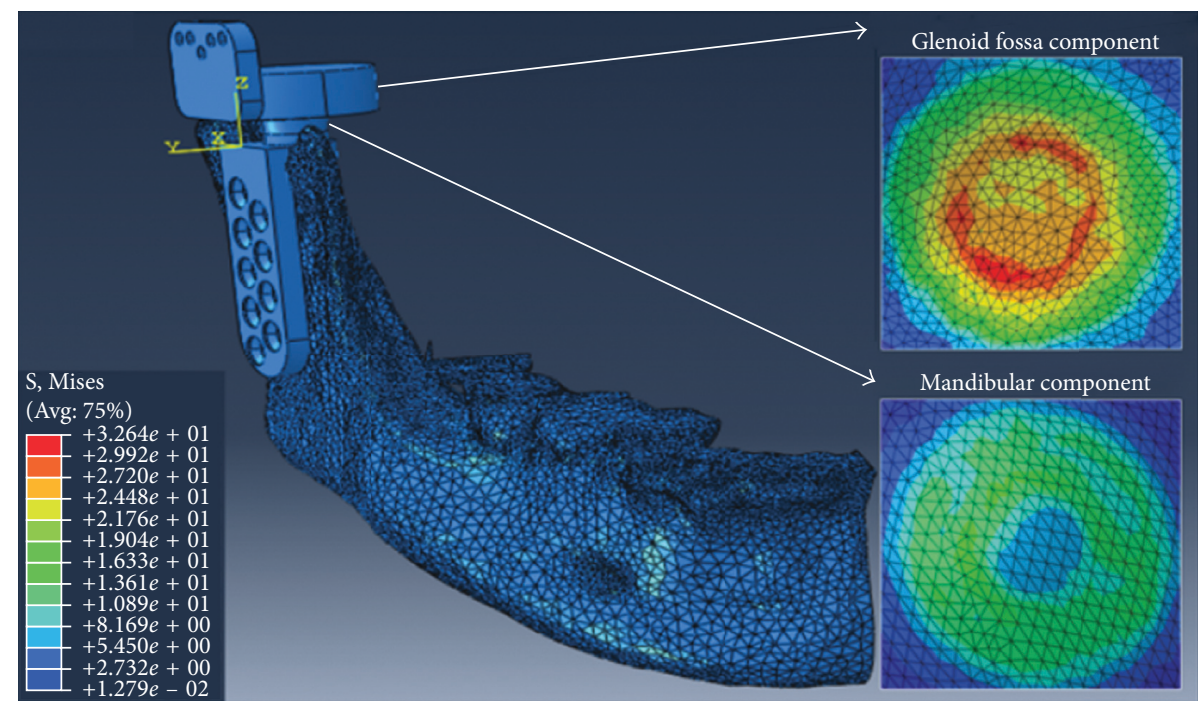

(c)

Figure 7: Contour of Mises contact stresses within the contact interface at the glenoid fossa and mandibular components for (a) cases 1, (b) 2 , and (c) 3 included in Tables 1 and 2. 
TABle 6: Maximum and average Mises equivalent stresses at the contact interface.

\begin{tabular}{lccc}
\hline Case & Component & $\sigma_{\text {eq,max }}(\mathrm{MPa})$ & $\sigma_{\text {eq,ave }}(\mathrm{MPa})$ \\
\hline \multirow{2}{*}{1} & Glenoid fossa & 1199.0 & 400.0 \\
& Mandibular & 699.4 & 250.0 \\
2 & Glenoid fossa & 149.4 & 74.8 \\
& Mandibular & 99.6 & 6.2 \\
\multirow{2}{*}{3} & Glenoid fossa & 32.6 & 26.1 \\
& Mandibular & 19.0 & 15.0 \\
\hline
\end{tabular}

components (such as Christensen's component) with customised glenoid fossa devices, being necessary the design and manufacturing of integrated prostheses, instead of those kinds of hybrid (half stock and half custom) implants.

Finally, the contact element equivalent stresses in case 3 (polymer on metal with a redesigned mandibular component) present a much more regular distribution of contact stresses. As can be seen in Figure 7(c), the stresses attained would be within the elastic regime, thus allowing a longer use of these kinds of medical devices without the kind of failure that has been reported in Section 4.1. Indeed, this is within the current trend for designing customised prostheses like Biomet-Lorenz and other new prototypes [21], which are already providing better results in terms of longevity and patients' comfort, as has been reported in recent research work $[13,17]$.

\section{Conclusions}

This paper presents a numerical analysis for the evaluation of contact stresses in different cases using generic designs of TMJ prostheses, including different materials and prosthesis geometries. Besides, the degradation of resected Christensen TMJ implants has been examined by microscope, and the damaged region is evaluated in terms of the possible mechanisms that could have promoted the wear and failure of the device.

This study based on numerical modelling and experimental evaluation of damaged zones at both TMJ prosthesis components (i.e., glenoid fossa and mandibular) has allowed establishing a link between the numerical results and experimental evidence. Indeed, the results show that the contact stresses attained in the interface between the mandibular and the glenoid fossa components, depending on the different prosthesis configurations considered, play a key role in the wear and subsequent failure of TMJR devices.

Finally, some specific contributions of this study must be remarked:

(i) A link between numerical results and experimental evidence of TMJR devices has been established. Indeed, although contact stresses within the interface are the key factor affecting wear and failure, it is also very important to examine resected prostheses in order to be able to establish design parameters and procedures.

(ii) Christensen TMJR configuration failure might not only be explained by wear under elastic regime due to tribocorrosion or fretting wear. It could also be due to a permanent deformation corresponding to an elastic-plastic behaviour within the TMJ prosthesis contact interface.

(iii) The numerical results obtained allow explaining the current trends of designing customised polymeron-metal TMJR devices with individualised shapes and softer (smaller radii) geometries.

\section{Conflicts of Interest}

The authors declare that they have no conflicts of interest.

\section{Acknowledgments}

The authors would like to thank Professor Antonio Ramos from the University of Aveiro and Professor Michel Mesnard from the University of Bordeaux for the valuable information provided and the interesting discussions that helped to improve this work.

\section{References}

[1] J. Ciurana, "Designing, prototyping and manufacturing medical devices: an overview," International Journal of Computer Integrated Manufacturing, vol. 27, no. 10, pp. 901-918, 2014.

[2] G. A. Tevelen, M. J. Pearcy, and R. W. Crawford, "Re-design of the Exeter V40 long-stem femoral component for ease of removal," Proceedings of the Institution of Mechanical Engineers, Part H: Journal of Engineering in Medicine, vol. 221, pp. 195-201, 2007.

[3] J. L. Maggs, A. Smeatham, S. L. Whitehouse, J. Charity, A. J. Timperley, and G. A. Gie, "The Exeter Contemporary flanged cemented acetabular component in primary total hip arthroplasty," The Bone and Joint Journal, vol. 98, no. 3, pp. 307-312, 2016.

[4] W. S. W. Harun, M. S. I. N. Kamariah, N. Muhamad, S. A. C. Ghani, F. Ahmad, and Z. Mohamed, "A review of powder additive manufacturing processes for metallic biomaterials," Powder Technology, vol. 327, pp. 128-151, 2018.

[5] P. K. Maji, A. J. Banerjee, P. S. Banerjee, and S. Karmakar, "Additive manufacturing in prosthesis development-a case study," Rapid Prototyping Journal, vol. 20, no. 6, pp. 480-489, 2014.

[6] A. A. A. Aliyu, A. M. Abdul-Rani, T. L. Ginta et al., "A review of additive mixed-electric discharge machining: current status and future perspectives for surface modification of biomedical implants," Advances in Materials Science and Engineering, vol. 2017, Article ID 8723239, 23 pages, 2017.

[7] R. Araújo, P. Teixeira, L. Montanari, A. Reis, M. B. Silva, and P. A. F. Martins, "Single point incremental forming of a facial implant," Prosthetics and Orthotics International, vol. 38, no. 5, pp. 369-378, 2014.

[8] G. Centeno, D. Morales-Palma, B. Gonzalez-Perez-Somarriba et al., "A functional methodology on the manufacturing of customized polymeric cranial prostheses from CAT using SPIF," Rapid Prototyping Journal, vol. 23, no. 4, pp. 771-780, 2017.

[9] I. Bagudanch, M. L. Garcia-Romeu, I. Ferrer, and J. Ciurana, "Customized cranial implant manufactured by incremental sheet forming using a biocompatible polymer," Rapid Prototyping Journal, 2017, In press. 
[10] L. M. Wolford, L. G. Mercuri, E. D. Schneiderman, R. Movahed, and W. Allen, "Twenty-year follow-up study on a patient-fitted temporomandibular joint prosthesis: the Techmedica/TMJ concepts device," Journal of Oral and Maxillofacial Surgery, vol. 73, no. 5, pp. 952-960, 2015.

[11] A. Kashi and R. W. Christensen, "Temporomandibular joint disorders: artificial joint replacements and future research needs," Journal of Long Term Effects of Medical Implants, vol. 16, pp. 459-474, 2006.

[12] H. E. Giannakopoulos, D. P. Sinn, and P. D. Quinn, "Biomet microfixation temporomandibular joint replacement system: a 3-year follow-up study of patients treated during 1995 to 2005," Journal of Oral and Maxillofacial Surgery, vol. 70, no. 4, pp. 787-794, 2012.

[13] L. M. Gonzalez-Perez, N. Fakih-Gomez, B. Gonzalez-PerezSomarriba, G. Centeno, and J. F. Montes-Carmona, "Twoyear prospective study of outcome following total temporomandibular joint replacement," International Journal of Oral and Maxillofacial Surgery, vol. 45, no. 1, pp. 78-84, 2016.

[14] M. Mathew, S. Kerwell, M. Alfaro, D. Royman, V. Barao, and S. Cortino, "Tribocorrosion and TMJ TJR devices," in Temporomandibular Joint Total Joint Replacement, Chapter 10, L. G. Mercuri, Ed., pp. 251-263, Springer International Publishing, Cham, Switzerland, 2016.

[15] A. N. Kanatas, C. Needs, A. B. Smith, A. Moran, G. Jenkins, and S. F. Worrall, "Short-term outcomes using the Christensen patient-specific temporomandibular joint implant system: a prospective study," British Journal of Oral and Maxillofacial Surgery, vol. 50, no. 2, pp. 149-153, 2012.

[16] A. Ramos and M. Mesnard, "Load transfer in Christensen TMJ in alloplastic total joint replacement for two different mouth apertures," Journal of Cranio-Maxillofacial Surgery, vol. 42, no. 7, pp. 1442-1449, 2014.

[17] L. M. Gonzalez-Perez, B. Gonzalez-Perez-Somarriba, G. Centeno, C. Vallellano, and J. F. Montes-Carmona, "Evaluation of total alloplastic temporo-mandibular joint replacement with two different types of prostheses: a threeyear prospective study," Medicina Oral Patología Oral y Cirugia Bucal, vol. 21, no. 6, pp. 766-775, 2016.

[18] M. Navarro, A. Michiardi, O. Castaño, and J. A. Planell, "Biomaterials in orthopaedics," Journal of The Royal Society Interface, vol. 5, pp. 1137-1158, 2010.

[19] M. Mesnard, J. C. Coutant, M. Aoun, J. Morlier, M. Cid, and P. Caix, "Relationships between geometry and kinematic characteristics in the temporomandibular joint," Computer Methods in Biomechanics and Biomedical Engineering, vol. 15, no. 4, pp. 393-400, 2011.

[20] M. Mesnard, A. Ramos, A. Ballu, J. Morlier, M. Cid, and J. A. Simoes, "Biomechanical analysis comparing natural and alloplastic temporomandibular joint replacement using a finite element model," Journal of Oral and Maxillofacial Surgery, vol. 69, no. 4, pp. 1008-1017, 2011.

[21] M. Mesnard and A. Ramos, "Towards a rigorous approach to designing a temporo mandibular joint prosthesis. From clinical challenge to numerical prototype," Procedia CIRP, vol. 5, pp. 141-146, 2013.

[22] ABAQUS/CAE Standard User's Manual, Version 6.12, Dassault Systèmes Simulia Corp., Providence, RI, USA, 2012.

[23] Z. Liu, Y. Fan, and Y. Qian, "Biomechanical simulation of the interaction in the temporomandibular joint within dentate mandible: a finite element analysis," in Porceedings of the IEEE/ICME International Conference on Complex Medical Engineering, pp. 1842-1846, Beijing, China, May 2007.
[24] A. Ramos, A. Completo, C. Relvas, M. Mesnard, and J. A. Simões, "Straight, semi-anatomic and anatomic TMJ implants: the influence of condylar geometry and bone fixation screws," Journal of Cranio-Maxillofacial Surgery, vol. 39, no. 5, pp. 343-350, 2011.

[25] J. F. Duque-Morán, R. Navarro-Navarro, R. Navarro-García, and J. A. Ruiz-Caballero, "Pares de fricción en la artroplastia total de cadera (ATC): Parámetros Tribológicos," Canarias Médica y Quirúrgica, vol. 9, no. 25, pp. 19-29, 2011.

[26] D. W. Hoeppner, V. Chandrasekaran, and C. Elliott, Fretting Fatigue: Current Technology and Practices, ASTM International, West Conshohocken, PA, USA, 2000.

[27] J. Vázquez, C. Navarro, and J. Domínguez, "Analysis of fretting fatigue initial crack path in Al7075-T651," Tribology International, vol. 108, pp. 87-94, 2017.

[28] R. B. Waterhouse, "Fretting wear," Wear, vol. 100, no. 1-3, pp. 107-118, 1984. 


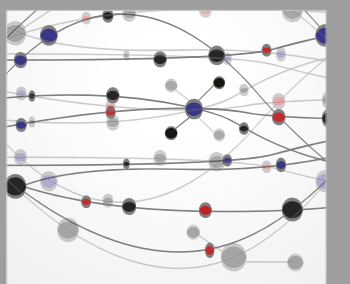

The Scientific World Journal
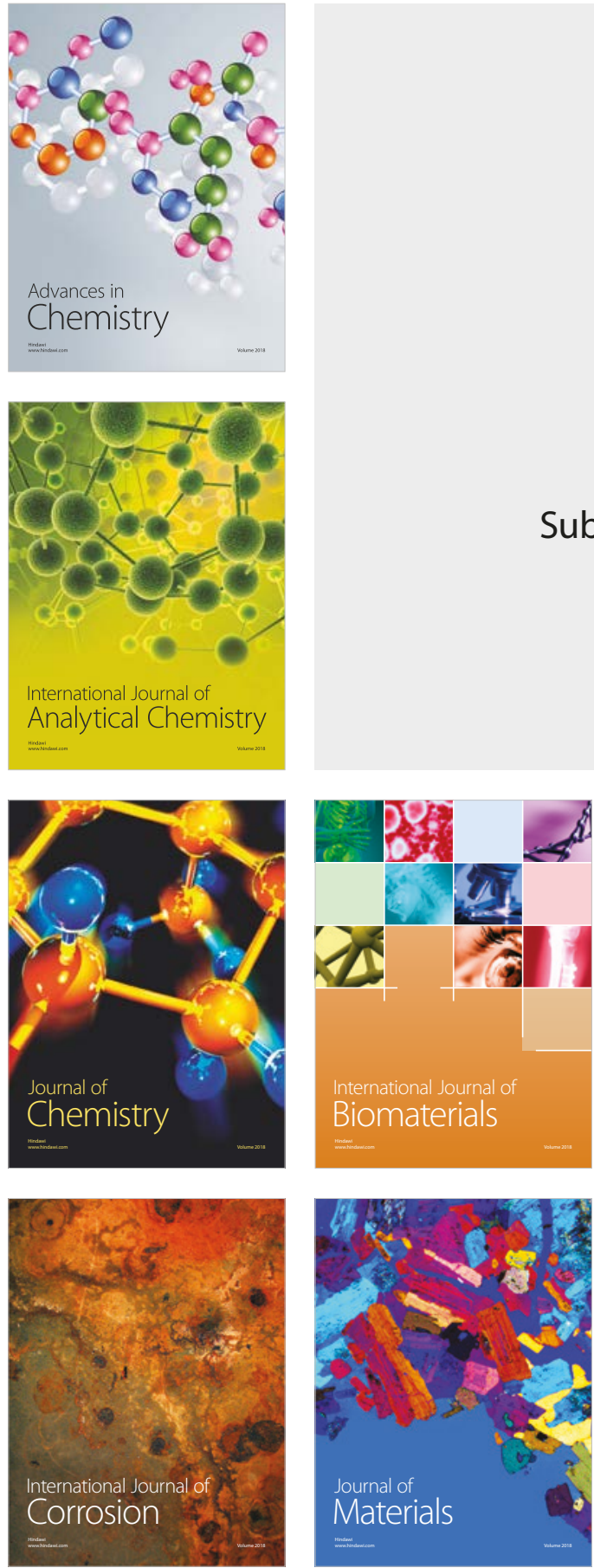

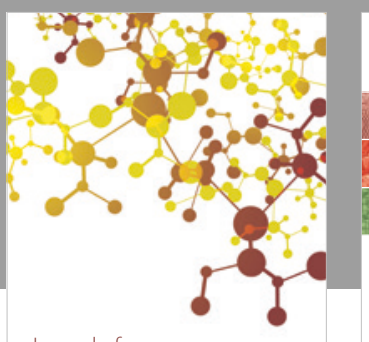

Journal of

Applied Chemistry
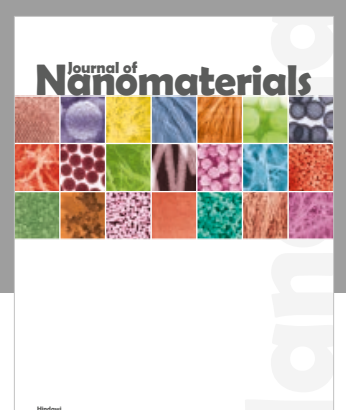

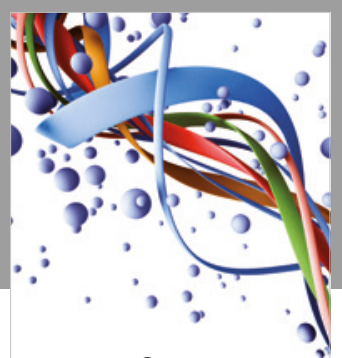

Scientifica

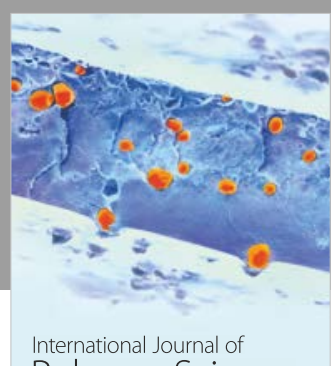

Polymer Science

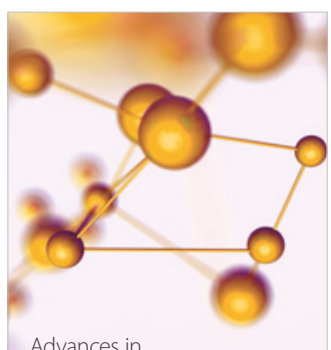

Physical Chemistry
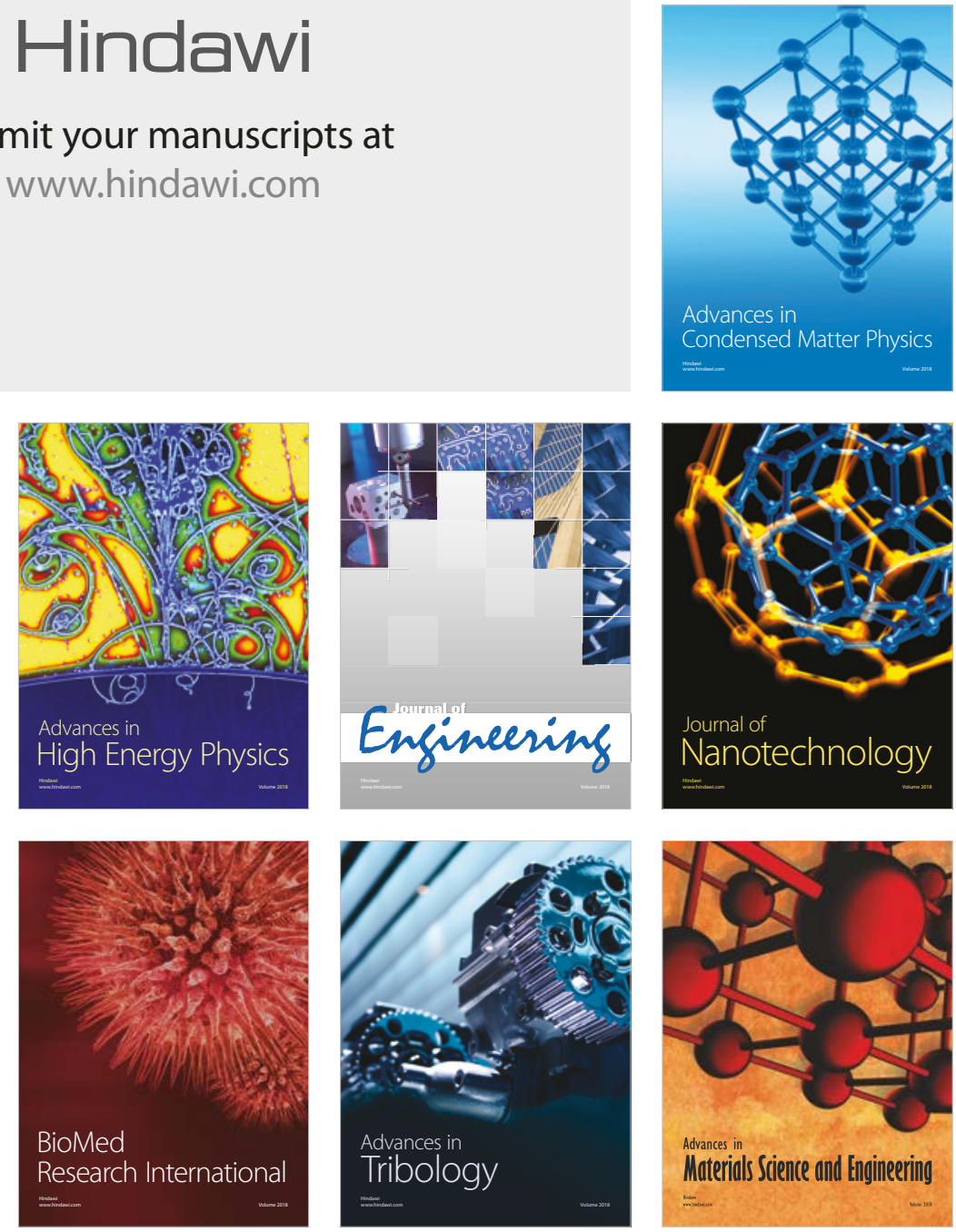